Check for updates

Cite this: Mater. Chem. Front. 2020, 4, 1492

Received 26th December 2019 Accepted 17th March 2020

DOI: 10.1039/c9qm00783k

rsc.li/frontiers-materials

\title{
From simple Katritzky salts to AlEgens: mechanochromic luminescence and heparin detection $\dagger$
}

\author{
Faxu Lin, ${ }^{\text {ab }}$ Yang Feng, ${ }^{a}$ Xiaoqing Liu, (D) ${ }^{c}$ Lei Wang, (D) ${ }^{a}$ Zhen-Qiang Yu (D) ${ }^{\text {bd }}$ and \\ Yi Liu (D)*ad
}

\begin{abstract}
Developing and exploring efficient fluorophores are of fundamental significance for sensing and tracking chemical or biological species and processes. Herein, a series of novel AlEgens derived from Katritzky pyridinium salts have been readily synthesized via a condensation reaction between their corresponding pyrylium precursors and versatile primary amines such as alkylamine and amino acid derivatives. The model compound TPP1 with a 2,4,6-triphenylpyridinium skeleton showed a smooth AIE curve without an inflection point, and the emission enhancement was attributed to the formation of water clusters around the pyridinium ring and the subsequent nano-aggregate formation, which hindered their intramolecular motions. Moreover, tetraphenylethene moiety-conjugated Katritzky salts retained classical AIE properties and were endowed with larger Stokes shifts and red-shifted emissions. Moreover, all these cationic AlE-active chromophores exhibited obvious mechanofluorochromism behaviors with redshifted luminescence upon grinding and blue-shifted emission after solvent annealing. The existence of the positively charged pyridinium ring endowed these luminogens with the capability to selectively detect Heparin in aqueous media from other ionic biopolymers.
\end{abstract}

\section{Introduction}

Owing to the capability of visualizing, tracking and evaluating invisible structures or processes within different dimensions and time scales ${ }^{1,2}$ fluorescence sensing and imaging have attracted tremendous attention. Large varieties of natural and synthetic fluorescent agents or probes, such as fluorescent proteins $^{3,4}$ and quantum dots, ${ }^{5,6}$ have been developed and applied as sensors or imaging agents. Among them, organic fluorophores are more advantageous as a result of their facile preparation, high efficiency for bioconjugation and labelling, ${ }^{7}$ and easy modulation of structures and performance via molecular engineering. ${ }^{8,9}$ However, most conventional organic

\footnotetext{
${ }^{a}$ Shenzhen Key Laboratory of Polymer Science and Technology, Guangdong Research Center for Interfacial Engineering of Functional Materials, College of Materials Science and Engineering, Shenzhen University, Shenzhen 518060, China. E-mail: liuyiacee@szu.edu.cn

${ }^{b}$ School of Chemistry and Environmental Engineering, Shenzhen University, Shenzhen 518060, China

${ }^{c}$ Shenzhen Grubbs Institute, Southern University of Science and Technology, Shenzhen 518005, China

${ }^{d}$ Centre for AIE Research, Shenzhen University, Shenzhen 518060, China

$\dagger$ Electronic supplementary information (ESI) available: Experimental details of synthesis and structural characterizations, cyclic voltammetry results, and other supplementary data. See DOI: 10.1039/c9qm00783k
}

luminogens comprise aromatic building blocks and have hydrophobic nature, which usually suffer from aggregationcaused quenching in aqueous media and biological systems. Therefore, the exploration of fluorophores with high emission efficiency in the aggregated state is still urgent.

Fortunately, a large family of luminogens featuring aggregation-induced emission (AIE) properties, ${ }^{10,11}$ which usually exhibit bright emission in aggregated or solid states, have been discovered and extensively explored in the past two decades. The typical AIE-active fluorophores, such as tetraphenylethene (TPE) ${ }^{12,13}$ are usually structurally featured with propeller-shaped and sterically congested geometry. The free intramolecular motions including the rotation and vibration of the AIE luminogens (AIEgens) will non-radiatively dissipate the excited-state energy and lead to low photoluminescence (PL) quantum yields. Upon aggregation, the restriction of intramolecular motions (RIMs) will block the non-radiative decay from the excited state, whereas the emission from AIEgens is boosted. Through delicate molecular engineering and material design, AIEgens have already been proven to be promising candidates for various applications, including fluorescence chemo/biosensing and imaging, ${ }^{14-19}$ diagnostics and therapeutics, ${ }^{20-22}$ and biomedicine. ${ }^{23,24}$ However, the development of new AIEgen systems is still urgent considering the following requirements: facile synthesis, easy conjugation to 
the target biomolecules, and easy structural screening for performance optimization.

Katritzky pyridinium salts, ${ }^{25-27}$ easily prepared in one step by the condensation of pyrylium salts with primary amines, are air and moisture-stable and convenient for purification via filtration. Recently, Katritzky salts have been widely investigated as precursors for the generation of alkyl radical species and the subsequent cross-coupling reactions..$^{28-30}$ Owing to the naturally abundant and inexpensive primary amines and bench-stable radical precursors, the Katritzky salts exhibit great synthetic potentials in organic synthesis. Among these salts, 1-alkyl-2,4,6-triphenylpyridinium salts are the most widely explored and studied due to the commercially available corresponding pyrylium salt precursors. The propeller-shaped geometry and highly free rotation of the phenyl rings within this series of Katritzky salts make them potential candidates for AIEgens. The abundance of primary amines in natural systems makes these fluorophores promising for the bio-labelling of biomolecules, especially proteins and glycans with $\mathrm{N}$-terminal residues. $^{31-33}$

Herein, we have synthesized an $N$-alkyl substituted Katritzky salt (TPP1 in Scheme 1) and investigated its PL behaviour in solvent mixtures. The classical AIEgen, namely TPE, was further conjugated onto the pyridinium salt skeleton to modulate the emission wavelength and other photophysical performances. Molecular engineering on the Katritzsk salt's skeleton was adjusted via a feasible choice of different pyrylium salt precursors, whereas a biologically abundant amino acid was also successfully attached to the fluorogenic backbone. This series of AIE-active pyridinium salts exhibited obvious mechanochromic luminescence (MCL) with greenish or yellow emission in the crystallized state and orange luminescence in the amorphous state. Additionally, the positively charged nature of these AIEgens

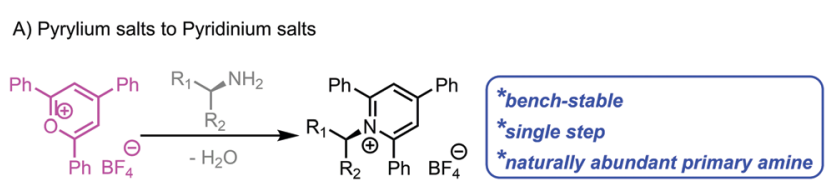

B) This work: novel AlEgens

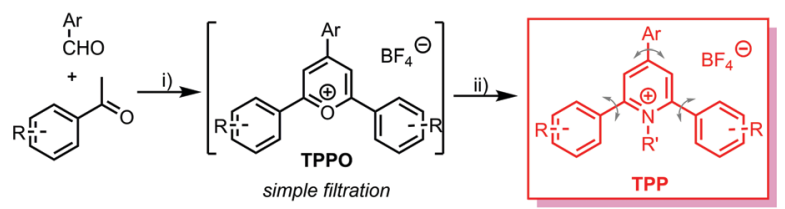

TPP1: $\mathrm{Ar}=\mathrm{Ph}, \mathrm{R}=$ para-Br, $\mathrm{R}^{\prime}=n-\mathrm{C}_{5} \mathrm{H}_{11} \quad$ TPP2: $\mathrm{Ar}=\mathrm{TPE}, \mathrm{R}=$ para- $\mathrm{Br}, \mathrm{R}^{\prime}=n-\mathrm{C}_{5} \mathrm{H}_{11}$

TPP3: $\mathrm{Ar}=\mathrm{TPE}, \mathrm{R}=$ para-Br, $\mathrm{R}^{\prime}=\mathrm{CH}_{2} \mathrm{COOMe} \mathrm{TPP} 4: \mathrm{Ar}=\mathrm{TPE}, \mathrm{R}=$ meta- $\mathrm{Br}, \mathrm{R}^{\prime}=n-\mathrm{C}_{5} \mathrm{H}_{11}$

TPP5: $\mathrm{Ar}=\mathrm{TPE}, \mathrm{R}=$ ortho- $\mathrm{Br}, \mathrm{R}^{\prime}=n-\mathrm{C}_{5} \mathrm{H}_{11} \quad$ TPP6: $\mathrm{Ar}=\mathrm{TPE}, \mathrm{R}=$ para-OMe, $\mathrm{R}^{\prime}=n-\mathrm{C}_{5} \mathrm{H}_{11}$

Scheme 1 (A) Facile synthesis of Katritzky pyridinium salts from the corresponding pyrylium precursors. (B) Synthetic routes toward novel AlEgens based on Katritzky salts. Conditions: (i) $\mathrm{BF}_{3} \cdot \mathrm{Et}_{2} \mathrm{O}$, toluene, $80{ }^{\circ} \mathrm{C}$, 46\% (TPPO1), 45\% (TPPO2), 38\% (TPPO3), 19\% (TPPO4), 50\% (TPPO5); (ii) primary amine, ethanol, $80{ }^{\circ} \mathrm{C}, 69 \%$ (TPP1), 92\% (TPP2), 86\% (TPP3), 83\% (TPP4), 66\% (TPP5), 98\% (TPP6). also enables them to selectively detect the biological polyelectrolyte, namely, heparin among other analogues. ${ }^{34,35}$

\section{Results and discussion}

\section{Synthesis of AIEgens}

Based on a previously reported protocol, pyrylium salt precursors, i.e., TPPOs (Scheme S1, ESI $\dagger$ ) were synthesized via a $\mathrm{BF}_{3} \cdot \mathrm{Et}_{2} \mathrm{O}$ promoted condensation reaction between benzaldehyde derivatives and acetophenone with various substitutions. The TPPO precursors were simply precipitated by pouring the reaction mixture into diethyl ether; they were obtained with satisfactory purity by filtration and used in the next step without further purification. Then, the reaction between the TPPO precursors and primary amines (including $n$-pentylamine and glycine methyl ester) readily yielded the targeted Katritzky salts (TPP in Scheme 1 and Scheme S1, ESI $\dagger$ ) after refluxing in ethanol. The cationic fluorophores could be purified by simple filtration or flash column chromatography with a high yield of around $60 \%$. The chemical identities of these novel luminogens were unambiguously confirmed by ${ }^{1} \mathrm{H}$ and ${ }^{13} \mathrm{C}$ nuclear magnetic resonance (NMR) spectroscopy. Mass spectra (Fig. S1-S6, ESI $\dagger$ ) of these compounds also showed the signals of the corresponding aromatic cations, which were well consistent with their molar masses.

\section{Aggregation-induced emission properties}

Prior to analysing Katritzky salts, the luminescence properties of their pyrylium precursors, namely, TPPOs were studied. The results indicated that all TPPOs emitted strongly in the solid state (Fig. S7, ESI $\dagger$ ), whereas the emission color ranged from yellow (545 $\mathrm{nm}$ for TPPO1) to red (730 $\mathrm{nm}$ for TPPO4). However, the PL experiment on TPPO2 revealed a new emission signal at $513 \mathrm{~nm}$, indicating the instability of the pyrylium salts in the DMSO- $\mathrm{H}_{2} \mathrm{O}$ mixture. As previously reported, ${ }^{36}$ the pyrylium ring is readily attacked by water to yield a cyclic hemiacetal intermediate, which would then decompose into a diketone product. The green emission observed in the PL measurement of TPPO2 was probably attributed to the TPE-substituted diketone species (Fig. S8, ESI $\dagger$ ). However, the bright emission of TPPOs in the solid state supported the possibility of its derivatives TPPs as novel AIE-active fluorophores.

The emission of TPP1 with the 2,4,6-triphenyl-pyridinium skeleton in a DMSO- $\mathrm{H}_{2} \mathrm{O}$ mixture was then investigated to verify whether this type of chromophore is AIE-active, and water was used as a non-solvent (Fig. 1 and Fig. S9, ESI $\dagger$ ). With the addition of water, the PL intensity at $460 \mathrm{~nm}$ continuously increased without a clear inflection point in the plots of relative fluorescence intensity $\left(I / I_{0}\right) v s$. water fraction $\left(f_{\mathrm{w}}\right)$. This phenomenon was in great contrast with that observed for other classical AIE molecules (e.g., silole, TPE or their derivatives), which only showed emission enhancement above a threshold water fraction owing to the formation of nano-aggregates. ${ }^{37-39}$ Similar to the result for the reported cationic 1,4-bis(4-pyridylethynyl)benzenebased macrocycle, ${ }^{40}$ the emission enhancement of TPP1 at a low 

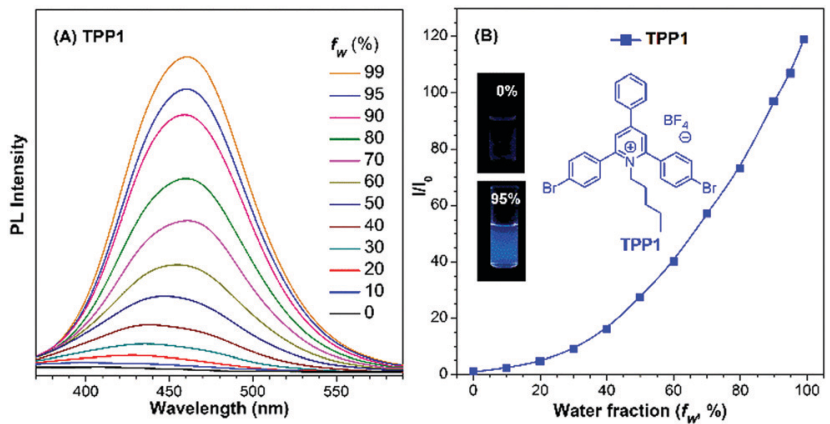

Fig. 1 (A) PL spectra of TPP1 in DMSO/water mixtures with different water fractions $\left(f_{\mathrm{w}}, \%\right)$. Excitation wavelength: $310 \mathrm{~nm}$ for TPP1. (B) Changes in the PL intensity (I) of TPP1 with different $f_{\mathrm{w}} \cdot l_{0}$ is the PL intensity of TPP1 at $460 \mathrm{~nm}$ in pure DMSO. Inset: Images of TPP1 under a UV lamp at $365 \mathrm{~nm}$ and its chemical structure. Concentration: $10 \mu \mathrm{M}$.

water fraction was probably attributed to the hydration of pyridinium cations and the formation of DMSO-water clusters in the mixture, ${ }^{41,42}$ which hampered the intramolecular rotation within the 2,4,6-triphenylpyridinium backbone and resulted in enhanced emission. Additionally, the emission maximum redshifted from $430 \mathrm{~nm}$ to $460 \mathrm{~nm}$. The bathochromic shift in the PL spectrum was probably owing to the increase in polarity with the increase in water fraction in the solvent mixture, which has already been investigated for similar solvatochromic dyes based on pyridinium salts. ${ }^{43}$

Dynamic light scattering (DLS) measurements also confirmed that nanoparticles were formed in the solvent mixture with a high water fraction, and the diameter of the aggregate was determined to be $178 \mathrm{~nm}$ for TPP1 in a DMSO/water mixture with $f_{\mathrm{w}}=95 \%$ (Fig. S10, ESI $\dagger$ ). This result also suggested the critical roles of aggregate formation and the subsequent restricted intramolecular motions for the emission enhancement for TPP1.

Compared with the PL intensity of the sample in pure DMSO, the PL intensity of TPP1 at $460 \mathrm{~nm}$ in a DMSO/water mixture with $f_{\mathrm{w}}=95 \%$ increased by approximately 110 -fold. The relative fluorescence quantum yield (QY) of TPP1 was measured to be $0.17 \%$ in pure DMSO and $24.9 \%$ in DMSO/water with $f_{\mathrm{w}}=$ $95 \%$, whereas the absolute QY of TPP1 was $2.5 \%$ in the asprepared solid state with the emission maximum at $418 \mathrm{~nm}$. These results evidently indicated that TPP1 was an AIE-active fluorophore. However, the QY and Stokes shift (approximately $150 \mathrm{~nm}$ ) of TPP1 in the solid state were found to be unsatisfactory. Moreover, the absorption maximum of TPP1 at around $310 \mathrm{~nm}$ inhibited the fluorophore to be excited by a common UV lamp (365 nm).

To further improve the PL performance of the Katritzky saltbased AIEgens and raise their Stokes shift, an extension of $\pi$-conjugation on the para-position of the pyridinium core was achieved. To avoid the detrimental ACQ impact of normal planar conjugation extension, the classical AIEgen, namely TPE, was attached to the para-position of the pyridinium ring for maintaining the AIE properties. Following this protocol, we designed and synthesized a series of Katritzky salt-type luminogens (from
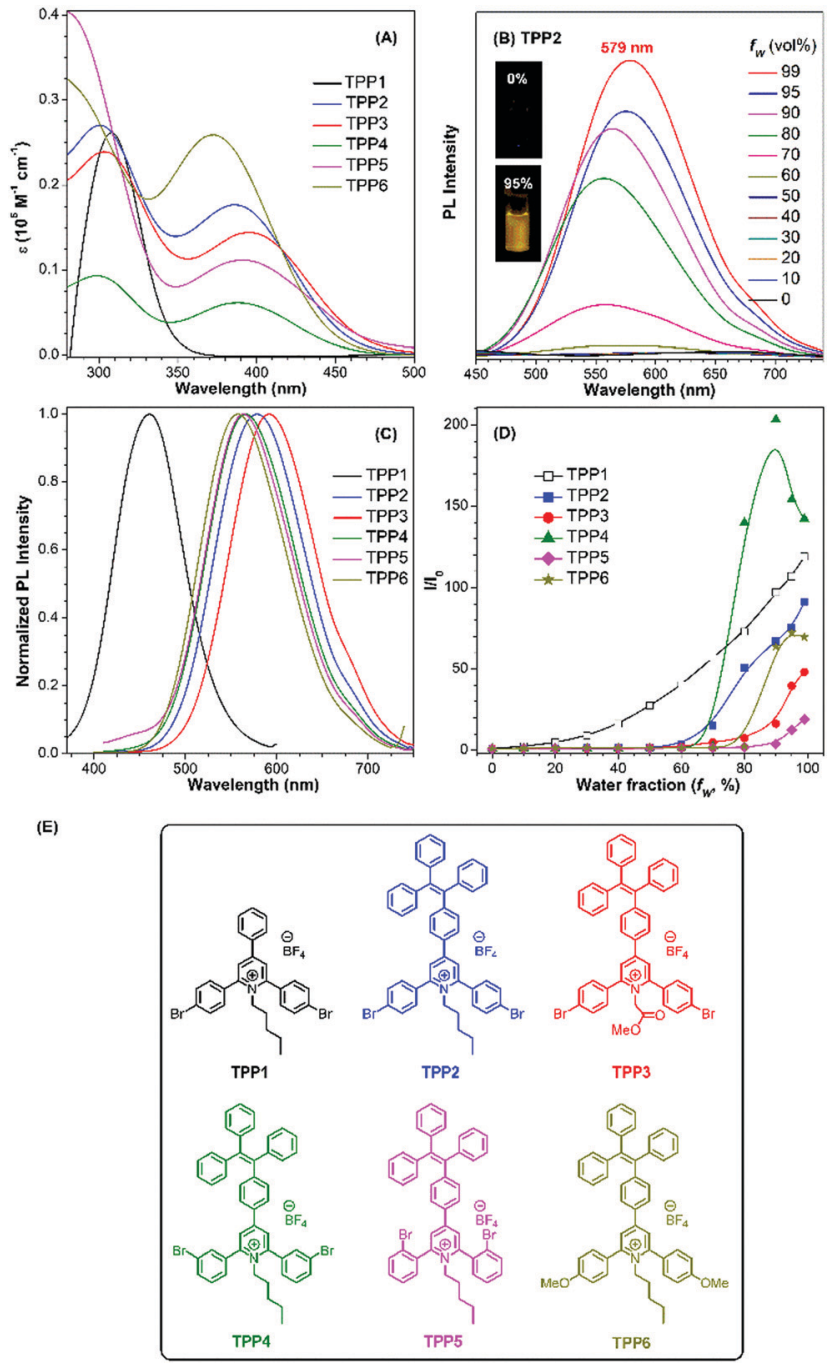

Fig. 2 (A) UV-Vis absorption spectra of TPPs in DMSO. (B) PL spectra of TPP2 in DMSO/water mixtures with different water fractions $\left(f_{\mathrm{w}}, \%\right)$; excitation wavelength: $390 \mathrm{~nm}$. Inset: Images of TPP2 under a UV lamp at $365 \mathrm{~nm}$. (C) Normalized PL spectra of TPPs in DMSO/water mixtures with different $f_{\mathrm{w}}$ at $99 \%$. Excitation wavelength: $310 \mathrm{~nm}$ for TPP1, $390 \mathrm{~nm}$ for TPP2 to TPP6. (D) Changes in the PL intensity (l) of TPPs with $f_{\mathrm{w}} \cdot l_{0}$ is the PL intensity of TPPs in pure DMSO. Concentration: $10 \mu \mathrm{M}$. (E) Chemical structures of TPPs.

TPP2 to TPP6) based on the condensation reaction between the corresponding pyrylium precursors and primary amines (including alkyl amine and amino acid derivatives) with satisfactory yields (Scheme S1, ESI $\dagger$ ). Additionally, their structures could be easily adjusted via modulating the substitution patterns on the 2- and 6-phenyl rings on the pyridinium ring and the nature of the substituents on the $\mathrm{N}$ atoms.

The UV-vis absorption and PL spectra of TPE-substituted pyridinium salts (TPP2 to TPP6, $10 \mu \mathrm{M}$ ) in DMSO/water mixtures were investigated with TPP1 used for comparison. As shown in Fig. 2a, all these fluorophores exhibit a maximum absorption peak at around $300 \mathrm{~nm}$ and a new absorption peak at around $390 \mathrm{~nm}$. For example, TPP2 showed two obvious absorption peaks at $300 \mathrm{~nm}$ and $386 \mathrm{~nm}$. The former peak, 
located close to the maximum absorption peak of TPP1 at $310 \mathrm{~nm}$, was owing to the triphenylpyridinium skeleton. The newly appeared absorption peak indicated the extension of $\pi$-conjugation and the creation of new chromophore skeletons. After replacing the $n$-pentyl group in TPP2 with natural amino acid derivatives, the absorption peaks of TPP3 slightly shifted to $304 \mathrm{~nm}$ and $397 \mathrm{~nm}$. Estimated from the absorption edge, the optical band gaps $\left(E_{\mathrm{g}}\right)$ of TPPs were $3.59,2.69,2.62,2.68,2.58$, and $2.77 \mathrm{eV}$. Hence, the $\pi$-extension with TPE moieties was proved to have a more significant impact on the electronic properties of TPPs (like $E_{\mathrm{g}}$ ) relative to the modulation of the substitution patterns on the 2- and 6-phenyl rings as well as the nature of the substituents on the $\mathrm{N}$ atoms.

The energetics of the frontier orbitals of TPPs were further investigated using cyclic voltammetry (Fig. S11, ESI $\dagger$ ). For all these fluorophores, no obvious oxidation peaks could be observed. From the onsets of the first reduction potential of TPPs relative to ferrocene, the energy levels of the lowest unoccupied molecular orbitals (LUMOs) of TPPs were estimated to be $-3.38,-3.37,-3.43,-3.41,-3.28$, and $-3.24 \mathrm{eV}$ (Fig. S12, ESI $\dagger$ ). Based on the $E_{\mathrm{g}}$ and LUMO levels, the highest occupied molecular orbitals (HOMOs) of TPPs were calculated to be $-6.97,-6.06,-6.05,-6.09,-5.86$, and $-6.01 \mathrm{eV}$. In comparison with the substitution patterns on the $\mathrm{N}$ atoms, the substitution patterns on the 2,6-phenyl rings and $\pi$-conjugation extension on the 4-phenyl ring of the pyridinium core had a more pronounced impact on the energy levels.

Subsequently, the PL spectra of TPE-substituted TPPs in the mixture of DMSO and water were investigated, and water was used as a poor solvent (Fig. 2, and Fig. S13-S17, ESI $\dagger$ ). As shown in Fig. 2d, all the TPE-conjugated Katritzky salts exhibit typical plots of $I / I_{0} v s$. $f_{\mathrm{w}}$ with clear inflection points and threshold $f_{\mathrm{w}}$ values for the enhancement in PL intensity. This phenomenon was well-consistent with most classical AIEgens, which indicated nano-aggregate formation as the dominant driving force for emission enhancement. For example, the luminogen TPP2, the structural analogue of TPP1, was nearly non-emissive with the $f_{\mathrm{w}}$ value below $50 \%$. On increasing the $f_{\mathrm{w}}$ value, the emission of TPP2 at $579 \mathrm{~nm}$ was gradually boosted owing to the formation of nano-aggregates (Fig. S13, ESI $\dagger$ ). DLS measurements implied the existence of nanoparticles of $114 \mathrm{~nm}$ diameter under $f_{\mathrm{w}}=95 \%$ (Fig. S10, ESI $\dagger$ ). Additionally, a slight redshift of $15 \mathrm{~nm}$ in the PL spectra was observed from $564 \mathrm{~nm}$ at $f_{\mathrm{w}}=90 \%$ to $579 \mathrm{~nm}$ at $f_{\mathrm{w}}=99 \%$, which was probably owing to the different self-organized micro-structures of TPP2 in the nano-aggregates.

After replacing the $n$-pentyl substituent with glycine methyl ester, the emission peak of TPP3 at $f_{\mathrm{w}}=99 \%$ further red-shifted to $592 \mathrm{~nm}$ (Fig. S14, ESI $\dagger$ ). Moreover, the constitutional isomers of TPP2, namely, TPP4 and TPP5 both exhibited strong emissions at $566 \mathrm{~nm}$ and $564 \mathrm{~nm}$, respectively (Fig. S15 and S16, ESI $\dagger$ ). On the other hand, TPP6 as the methoxy groupsubstituted analogue emitted at $558 \mathrm{~nm}$ (Fig. S17, ESI $\dagger$ ). The absolute QY of all these TPE-substituted TPPs in pure DMSO was in the range from $3 \%$ to $4 \%$. After nano-aggregate formation, the QY values of all these luminogens increased significantly. For instance, the absolute QY of TPP2 at $f_{\mathrm{w}}=95 \%$ and the solid increased to $50.3 \%$ and $45.5 \%$, respectively. Additionally, the fluorescence lifetime of TPE-substituted TPPs obviously increased after the formation of nano-aggregates (Fig. S18, ESI $\dagger$ ). This enhancement reflected TPPs being in a more restricted state in the aggregated state, which was consistent with the restricted intramolecular motion mechanism for AIEgens. Hence, the AIE property and large Stokes shift with the long-wavelength emission of TPE-substituted Katritzky salts were both achieved in these new AIEgens, as summarized in Table S1 (ESI $\dagger$ ).

\section{Mechanochromic luminescence}

Regarding the highly twisted conformations of TPPs, the MCL properties of these cationic luminogens were also investigated. As shown in Fig. 3, when the as-prepared TPP1 sample is ground into a powder with a mortar and pestle, the emission maximum shifts from $418 \mathrm{~nm}$ for the as-prepared sample to $426 \mathrm{~nm}$ after grinding. Then, the ground powder was put into a sealed beaker containing a small amount of acetone with bubbling argon gas for 5 minutes at room temperature; consequently, the emission wavelength of TPP1 reverted back to $422 \mathrm{~nm}$.

To further elucidate the underlying reasons for the MCL behavior of cationic fluorophores, powder X-ray diffraction (XRD) was carried out for the samples to study the changes in molecular arrangements in the solid state. For TPP1, the powder XRD patterns of the as-prepared samples showed two broad diffraction peaks at around $12^{\circ}$ and $20^{\circ}$ in the wide-angle region, which indicated the amorphous structures and loose packing of fluorophores. After grinding, the diffraction peak at $20^{\circ}$ was further attenuated, whereas the peak at $12^{\circ}$ completely disappeared. This result implied a further decrease in the arrangement order in the ground sample, leading to a slightly red-shifted emission to $426 \mathrm{~nm}$. After annealing the ground sample with acetone vapour, the diffraction pattern remained unchanged and was almost identical to that of the ground sample; hence, the luminescence only recovered back to $422 \mathrm{~nm}$.

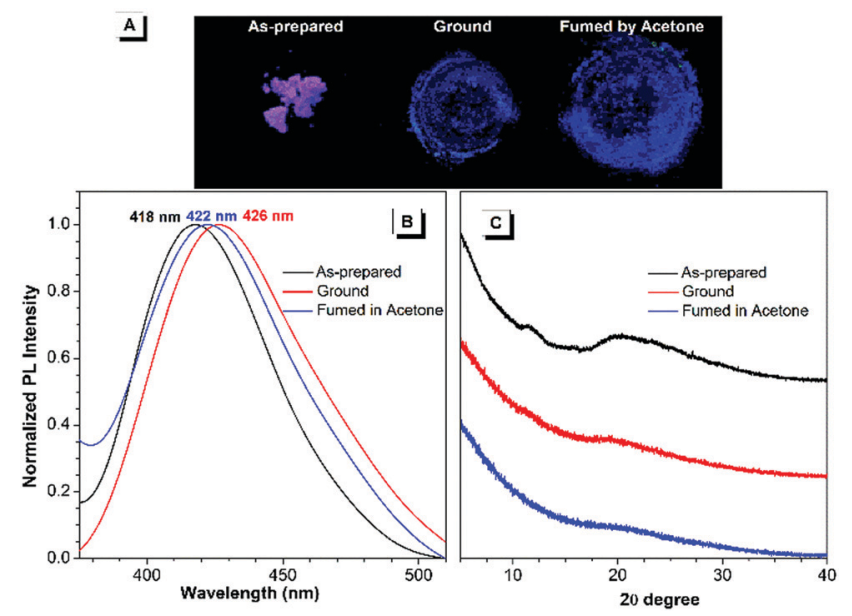

Fig. 3 Fluorescence images (A), PL spectra (B) and powder XRD results (C) of the as-prepared TPP1 samples after grinding and subsequent solvent fuming with acetone for $5 \mathrm{~min}$. 

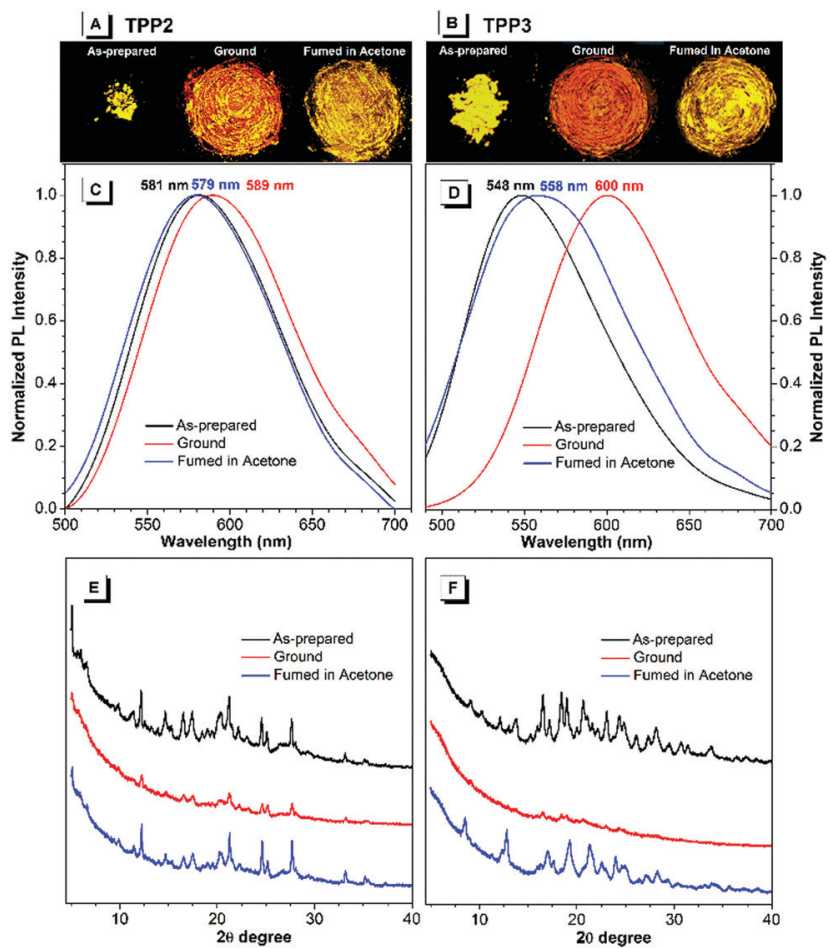

Fig. 4 Fluorescence images, PL spectra, and powder XRD patterns of the as-prepared samples of the cationic luminogen TPP2 (A), (C), (E) and TPP3 (B), (D) and (F) after grinding and subsequent solvent fuming with acetone for $5 \mathrm{~min}$.

To investigate the conformations and packing models of TPP1 in the solid state, efforts to obtain the X-ray crystal structure of TPP1 were extensively made under different conditions. Unfortunately, crystallization experiments with this cationic fluorophore failed to yield suitable single crystals for $\mathrm{X}$-ray analysis. The lack of appropriate crystallization capability of TPP1 also resulted in its subtle emission change upon mechanical stimulus and solvent annealing.

By contrast, the TPE-substituted Katritzky salts exhibited more pronounced MCL phenomena. After grinding the pristine samples of TPP2, the emission red-shifted from 581 to $589 \mathrm{~nm}$, and the emission wavelength could recover back to $579 \mathrm{~nm}$ after annealing with acetone vapour for 5 minutes (Fig. 4). The powder XRD results of the as-prepared TPP2 samples showed a series of sharp diffraction signals, indicating a highly crystalline and densely packed structure. After vigorous grinding, a similar XRD pattern with most peaks either disappearing or attenuating was recorded, suggesting a more loosely organized structure of TPP2 in the solid state. This decrease in the packing order for TPP2 resulted in slightly red-shifted emission from 581 to $589 \mathrm{~nm}$. After fuming with acetone vapor, a series of sharp diffraction peaks were recovered in the XRD patterns, indicating the formation of highly ordered structures and the obvious blue-shifted emission at $579 \mathrm{~nm}$.

Similarly, the methylated glycine-tailed cationic luminogen, namely, TPP3 exhibited more pronounced MCL behavior. The PL spectra of the as-prepared samples showed a remarkable shift from $548 \mathrm{~nm}$ to $600 \mathrm{~nm}$ after grinding, and this was then restored to $558 \mathrm{~nm}$ after solvent annealing (Fig. 4). The XRD patterns of the pristine and annealed samples showed identical series of sharp diffraction peaks, whereas all the peaks in the small-angle region completely disappeared for the ground sample. Therefore, similar to typical AIEgens with MCL properties, the transformation between the crystallized and amorphous states was the driving force for the MCL process of these cationic luminogens. In the crystalized structure, dense packing of AIEgens enforced the molecules to adapt a more congested conformation, and the twisting of aryl rings for better fitting into the crystalline lattices led to bluer emission for the crystals of AIEgens. By contrast, the molecules in the amorphous state would assume a more planar conformation and thus show redder emission without constraints from lattices. ${ }^{4,45}$

During the MCL process of AIEgens, the amplitude of emission changes is mainly determined by the crystallization capabilities and the conformation difference of the molecules between the crystallized and amorphous structures. For TPP1 and TPP4, the small emission changes after grinding were mainly attributed to their lack of crystallization capabilities, which were supported by their XRD patterns after solvent annealing (Fig. S19, ESI $\dagger$ ). The other three TPPs, namely, TPP3, TPP5, and TPP6 exhibited significant shifts of approximately 50, 35, and $60 \mathrm{~nm}$, respectively, whereas a small shift of approximately $10 \mathrm{~nm}$ was observed for TPP2 during MCL experiments (Fig. 4 and Fig. S20 and S21, ESI $\dagger$ ). However, these four fluorophores showed sharp and intense diffractions for pristine and annealed samples with faint signals after grinding. Therefore, the detailed amplitude of the conformational transformation of TPPs in the crystal lattices and amorphous solids probably contributed to the variations in the MCL phenomena for these similar AIEgens. These results also illustrated the critical impacts of substitution patterns both at the phenyl rings and $\mathrm{N}$ atoms on the photophysical properties of AIEgens in the solid state.

\section{Heparin detections}

As cationic luminogens, TPPs were structurally featured with positively charged backbones, which endowed these molecules with native interaction capabilities with anionic molecules and biomacromolecules via Coulombic force in an aqueous solution. The formation of a TPP/biopolymer complex would restrict the intramolecular motions of TPPS and consequently turn on the emission of the TPPs units. ${ }^{46,47}$ Herein, we explored their possibilities of detecting anionic biopolymers in aqueous mixtures, including heparin (Hep), chondroitin 4-sulfate (Chs), and hyaluronic acid (HA). The concentration of biopolymers was calculated using repeating disaccharides as the monomer units.

First, we investigated the fluorescence response of TPP1 in aqueous mixtures to biopolymers. As shown in Fig. 5, on increasing the concentration of polysaccharides, the fluorescence intensity of TPP1 remains unchanged even after the addition of $60 \mu \mathrm{M}$ biopolymers (Fig. S22, ESI $\dagger$ ). By contrast, all TPE-substituted TPPs exhibited remarkable emission enhancements after the addition of Hep, which moderately 

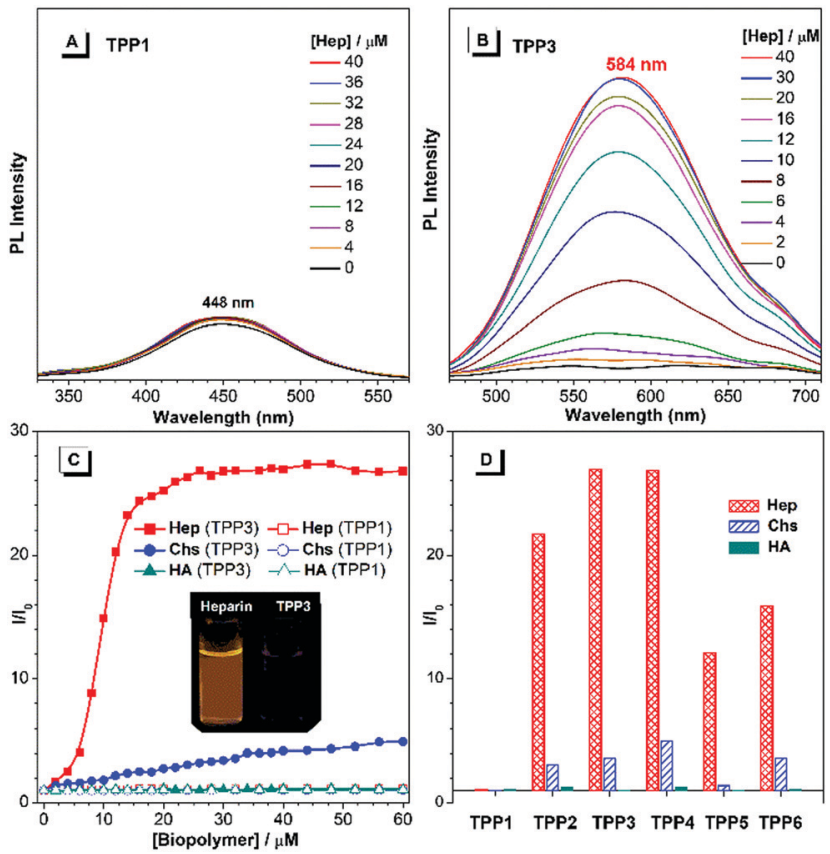

Fig. 5 PL spectra of TPP1 (A) and TPP3 (B) with different concentrations of heparin. (C) Curve of relative fluorescence intensity of TPP1 and TPP3 with biopolymer concentration. Inset: Fluorescence photos of TPP3 in the absence or presence of different biopolymers $(40 \mu \mathrm{M})$. (D) PL response of TPPs in the presence of different biopolymers $(40 \mu \mathrm{M})$. Solvent: DMSO/ $\mathrm{H}_{2} \mathrm{O}=50 / 50$ by volume; concentration of cationic AlEgens: $10 \mu \mathrm{M}$.

increased with the addition of Chs with almost no dependence on the concentration of HA (Fig. 5, and Fig. S23-S27, ESI $\dagger$ ). This selectivity was directly correlated with the density of negative charges on the poly-saccharide backbones. ${ }^{48}$ Heparin has four ionic groups on the disaccharide monomer unit, whereas only two and one single ionic sites are located at the monomer moieties of Chs and HA, respectively. Consequently, TPPs exhibited the strongest electrostatic interactions with heparin, moderate complexation with Chs, and weak bonding with HA. Owing to the small size and hydration shell of TPP1 in the DMSO/ $\mathrm{H}_{2} \mathrm{O}$ mixture, the interaction between TPP1 and biopolymers was strongly attenuated, leading to nearly no emission changes after adding biopolymers.

The PL spectra of TPP3, namely, the amino acid derivativetailed pyridinium salt showed rapidly increasing emission at $584 \mathrm{~nm}$ after the addition of Hep in the mixture of DMSO and water $\left(f_{\mathrm{w}}=50 \%\right)$. The PL intensity approached a plateau and showed emission enhancement of about 26-fold when the concentration of Hep reached $24 \mu \mathrm{M}$ (Fig. S24, ESI $\dagger$ ). However, on further increasing the concentration of Hep up to $60 \mu \mathrm{M}$, the PL spectra remained almost identical. By contrast, the emission of TPP3 increased smoothly and almost linearly with the addition of Chs. The PL intensity at $584 \mathrm{~nm}$ was enhanced by approximately 4 -fold when the concentration of Chs was increased to $40 \mu \mathrm{M}$. The addition of HA exhibited nearly no influence on the emission of the fluorophore. For other analogues, the response behaviour to polysaccharides followed a similar manner. The response range of TPPs (from TPP2 to
TPP6) to Hep typically from $0 \mu \mathrm{M}$ tp $14 \mu \mathrm{M}$ fully covered the whole clinical dose range of Hep $(1.7-10 \mu \mathrm{M}),{ }^{49}$ which made these easily accessible and sensitive AIEgens highly promising for clinical applications.

\section{Conclusions}

In summary, a series of cationic fluorophores (TPPs) based on Katritzky pyridinium backbones were readily synthesized by the condensation of the corresponding pyrylium salts and versatile primary amines including amino acid derivatives. The free rotation of phenyl rings within the 2,4,6-triphenylpyridinium framework endowed the pristine Katritzky salts with obvious AIE properties. On increasing the water fraction in the DMSO/ water mixture, the emission of TPP1 was remarkably enhanced without a clear inflection point observed in its PL spectra. This intriguing type of AIE phenomenon was probably owing to the formation of water clusters around the pyridinium sites via hydrogen bonding, which further restricted the intramolecular rotations of the phenyl rings within the Katritzky salts. Additionally, substitution engineering of Katritzky salts was accomplished to modulate their photophysical properties, including $\pi$-conjugation extension with the TPE units, substitution engineering on the 2,6-phenyl rings, and chemical conjugation with diverse primary amines. The mechanochromic luminescence behaviour of these cationic luminogens was further explored in the solid state as a result of their different molecular packing models and conformations in amorphous and crystallized structures. Moreover, the emission of these cationic AIEgens in an aqueous mixture was found to be selectively "turned on" by heparin due to the electrostatic force between pyridinium sites and polysaccharide backbones. These new cationic AIEgens can facilitate the synthetic exploration of new molecular structures with AIE properties and also provide an approach for fluorescence labelling on amine group-containing biomacromolecules, especially peptides and proteins.

\section{Conflicts of interest}

There are no conflicts to declare.

\section{Acknowledgements}

This work is partially supported by the National Natural Science Foundation of China $(21704065,21674065)$, the Innovation Research Foundation of Shenzhen (JCYJ20170817100547775) and Shenzhen University (No. 2018005). We thank the Instrumental Analysis Centre of Shenzhen University (Xili Campus) for NMR measurement.

\section{Notes and references}

$1 \mathrm{~V}$. Ntziachristos, Fluorescence molecular imaging, Аппи. Rev. Biomed. Eng., 2006, 8, 1. 
2 T.-L. Liu, S. Upadhyayula, D. E. Milkie, V. Singh, K. Wang, I. A. Swinburne, K. R. Mosaliganti, Z. M. Collins, T. W. Hiscock, J. Shea, A. Q. Kohrman, T. N. Medwig, D. Dambournet, R. Forster, B. Cunniff, Y. Ruan, H. Yashiro, S. Scholpp, E. M. Meyerowitz, D. Hockemeyer, D. G. Drubin, B. L. Martin, D. Q. Matus, M. Koyama, S. G. Megason, T. Kirchhausen and E. Betzig, Observing the cell in its native state: Imaging subcellular dynamics in multicellular organisms, Science, 2018, 360, eaaq1392.

3 R. Y. Tsien, Constructing and Exploiting the Fluorescent Protein Paintbox (Nobel Lecture), Angew. Chem., Int. Ed., 2009, 48, 5612.

4 D. S. Bindels, L. Haarbosch, L. van Weeren, M. Postma, K. E. Wiese, M. Mastop, S. Aumonier, G. Gotthard, A. Royant, M. A. Hink and T. W. J. Gadella, mScarlet: a bright monomeric red fluorescent protein for cellular imaging, Nat. Methods, 2017, 14, 53.

5 X. Michalet, F. F. Pinaud, L. A. Bentolila, J. M. Tsay, S. Doose, J. J. Li, G. Sundaresan, A. M. Wu, S. S. Gambhir and S. Weiss, Quantum Dots for Live Cells, in Vivo Imaging, and Diagnostics, Science, 2005, 307, 538.

6 O. T. Bruns, T. S. Bischof, D. K. Harris, D. Franke, Y. Shi, L. Riedemann, A. Bartelt, F. B. Jaworski, J. A. Carr, C. J. Rowlands, M. W. B. Wilson, O. Chen, H. Wei, G. W. Hwang, D. M. Montana, I. Coropceanu, O. B. Achorn, J. Kloepper, J. Heeren, P. T. C. So, D. Fukumura, K. F. Jensen, R. K. Jain and M. G. Bawendi, Next-generation in vivo optical imaging with short-wave infrared quantum dots, Nat. Biomed. Eng., 2017, 1, 0056.

7 M. S. T. Gonçalves, Fluorescent Labeling of Biomolecules with Organic Probes, Chem. Rev., 2009, 109, 190.

8 D. W. Domaille, E. L. Que and C. J. Chang, Synthetic fluorescent sensors for studying the cell biology of metals, Nat. Chem. Biol., 2008, 4, 168.

9 C. Zhu, L. Liu, Q. Yang, F. Lv and S. Wang, Water-Soluble Conjugated Polymers for Imaging, Diagnosis, and Therapy, Chem. Rev., 2012, 112, 4687.

10 Y. Hong, J. W. Y. Lam and B. Z. Tang, Aggregation-induced emission, Chem. Soc. Rev., 2011, 40, 5361.

11 J. Mei, N. L. C. Leung, R. T. K. Kwok, J. W. Y. Lam and B. Z. Tang, Aggregation-Induced Emission: Together We Shine, United We Soar!, Chem. Rev., 2015, 115, 11718.

12 Y. Liu, F. X. Lin, Y. Feng, X. Liu, L. Wang, Z.-Q. Yu and B. Z. Tang, Shape-Persistent $\pi$-Conjugated Macrocycles with Aggregation-Induced Emission Property: Synthesis, Mechanofluorochromism, and Mercury(II) Detection, ACS Appl. Mater. Interfaces, 2019, 11, 34232.

13 Y. Liu, L. H. You, F. X. Lin, K. Fu, W. Z. Yuan, E.-Q. Chen, Z.-Q. Yu and B. Z. Tang, Highly Efficient Luminescent Liquid Crystal with Aggregation-Induced Energy Transfer, ACS Appl. Mater. Interfaces, 2019, 11, 3516.

14 Y. Liu, C. Deng, L. Tang, A. Qin, R. Hu, J. Z. Sun and B. Z. Tang, Specific Detection of D-Glucose by a Tetraphenylethene-Based Fluorescent Sensor, J. Am. Chem. Soc., 2011, 133, 660.

15 M. Gao and B. Z. Tang, Fluorescent Sensors Based on Aggregation-Induced Emission: Recent Advances and Perspectives, ACS Sens., 2017, 2, 1382.
16 D. D. La, S. V. Bhosale, L. A. Jones and S. V. Bhosale, Tetraphenylethylene-Based AIE-Active Probes for Sensing Applications, ACS Appl. Mater. Interfaces, 2018, 10, 12189.

$17 \mathrm{~J}$. Mei, Y. Huang and H. Tian, Progress and Trends in AIEBased Bioprobes: A Brief Overview, ACS Appl. Mater. Interfaces, 2018, 10, 12217.

18 J.-S. Ni, H. Liu, J. Liu, M. Jiang, Z. Zhao, Y. Chen, R. T. K. Kwok, J. W. Y. Lam, Q. Peng and B. Z. Tang, The unusual aggregation-induced emission of coplanar organoboron isomers and their lipid droplet-specific applications, Mater. Chem. Front., 2018, 2, 1498.

19 J. Tavakoli, H.-p. Zhang, B. Z. Tang and Y. Tang, Aggregation-induced emission lights up the swelling process: a new technique for swelling characterisation of hydrogels, Mater. Chem. Front., 2019, 3, 664.

20 D. Wang, M. M. S. Lee, W. Xu, R. T. K. Kwok, J. W. Y. Lam and B. Z. Tang, Theranostics based on AIEgens, Theranostics, 2018, 8, 4925.

21 Y.-L. Wang, C. Fan, B. Xin, J.-P. Zhang, T. Luo, Z.-Q. Chen, Q.-Y. Zhou, Q. Yu, X.-N. Li, Z.-L. Huang, C. Li, M.-Q. Zhu and B. Z. Tang, AIE-based super-resolution imaging probes for $\beta$-amyloid plaques in mouse brains, Mater. Chem. Front., 2018, 2, 1554.

22 M. M. S. Lee, L. Zheng, B. Yu, W. Xu, R. T. K. Kwok, J. W. Y. Lam, F. Xu, D. Wang and B. Z. Tang, A highly efficient and AIE-active theranostic agent from natural herbs, Mater. Chem. Front., 2019, 3, 1454.

23 C. Zhu, R. T. K. Kwok, J. W. Y. Lam and B. Z. Tang, Aggregation-Induced Emission: A Trailblazing Journey to the Field of Biomedicine, ACS Appl. Bio Mater., 2018, 1, 1768.

24 D. Ding, in Principles and Applications of Aggregation-Induced Emission, ed. Y. Tang and B. Z. Tang, Springer International Publishing, Cham, 2019, p. 457.

25 A. R. Katritzky and S. S. Thind, The synthesis and reactions of sterically constrained pyrylium and pyridinium salts, J. Chem. Soc., Perkin Trans. 1, 1980, 1895.

26 A. R. Katritzky, N. E. Grzeskowiak, N. F. Eweiss and E. A. Elsherbini, Triphenylpyridinium derivatives of $\alpha$-amino-acids and a dipeptide, J. Chem. Soc., Perkin Trans. 1, 1983, 497.

27 A. R. Katritzky, M. De Rosa and N. E. Grzeskowiak, Pyryliummediated transformations of natural products. Part 1. Synthesis and hydrolysis of 4-(4-methoxy-3-sulphophenyl)-2,6-bis(4-sulphophenyl) pyrylium perchlorate: a new water-soluble pyrylium cation, J. Chem. Soc., Perkin Trans. 2, 1984, 841.

28 F. J. R. Klauck, M. J. James and F. Glorius, Deaminative Strategy for the Visible-Light-Mediated Generation of Alkyl Radicals, Angew. Chem., Int. Ed., 2017, 56, 12336.

29 D. Kong, P. J. Moon and R. J. Lundgren, Radical coupling from alkyl amines, Nat. Catal., 2019, 2, 473.

30 J. Wu, P. S. Grant, X. Li, A. Noble and V. K. Aggarwal, Catalyst-Free Deaminative Functionalizations of Primary Amines by Photoinduced Single-Electron Transfer, Angew. Chem., Int. Ed., 2019, 58, 5697.

31 M. H. O'Leary and G. A. Samberg, Chemical modification of proteins by pyrylium salts, J. Am. Chem. Soc., 1971, 93, 3530. 
32 S. A. Johannesen, S. R. Beeren, D. Blank, B. Y. Yang, R. Geyer and $\mathrm{O}$. Hindsgaul, Glycan analysis via derivatization with a fluorogenic pyrylium dye, Carbohydr. Res., 2012, 352, 94.

33 M. Waliczek, M. Kijewska, M. Rudowska, B. Setner, P. Stefanowicz and Z. Szewczuk, Peptides Labeled with Pyridinium Salts for Sensitive Detection and Sequencing by Electrospray Tandem Mass Spectrometry, Sci. Rep., 2016, 6, 37720.

34 Y. Huang, G. Zhang, F. Hu, Y. Jin, R. Zhao and D. Zhang, Emissive nanoparticles from pyridinium-substituted tetraphenylethylene salts: imaging and selective cytotoxicity towards cancer cells in vitro and in vivo by varying counter anions, Chem. Sci., 2016, 7, 7013.

35 L. Zhang, L. Jiao, J. Zhong, W. Guan and C. Lu, Lighting up the interactions between bacteria and surfactants with aggregation-induced emission characteristics, Mater. Chem. Front., 2017, 1, 1829.

36 A. Williams, Hydrolysis of pyrylium salts. Kinetic evidence for hemiacetal intermediates, J. Am. Chem. Soc., 1971, 93, 2733.

37 J. Jia and H. Zhao, Remarkable isomeric effects on the mechanofluorochromism of tetraphenylethylene-based D- $\pi$-A derivatives, New J. Chem., 2019, 43, 2231.

38 Z. Lu, S. Yang, X. Liu, Y. Qin, S. Lu, Y. Liu, R. Zhao, L. Zheng and $H$. Zhang, Facile synthesis and separation of $E / Z$ isomers of aromatic-substituted tetraphenylethylene for investigating their fluorescent properties via single crystal analysis, J. Mater. Chem. C, 2019, 7, 4155.

39 T. S. Reddy, S. Lee and M. S. Choi, Position and conjugation-dependent aggregation-induced emission enhancement properties of naphthalimide-tetraphenylethylene conjugates, Dyes Pigm., 2019, 168, 49.

40 Z. Wang, W. Bai, J. Tong, Y. J. Wang, A. Qin, J. Z. Sun and B. Z. Tang, A macrocyclic 1,4-bis(4-pyridylethynyl)benzene showing unique aggregation-induced emission properties, Chem. Commun., 2016, 52, 10365.
$41 \mathrm{H}$. Ohtaki and T. Radnai, Structure and dynamics of hydrated ions, Chem. Rev., 1993, 93, 1157.

42 N. Engel, K. Atak, K. M. Lange, M. Gotz, M. Soldatov, R. Golnak, E. Suljoti, J.-E. Rubensson and E. F. Aziz, DMSO-Water Clustering in Solution Observed in Soft X-ray Spectra, J. Phys. Chem. Lett., 2012, 3, 3697.

43 C. Reichardt, Solvatochromic Dyes as Solvent Polarity Indicators, Chem. Rev., 1994, 94, 2319.

44 Y. Q. Dong, J. W. Y. Lam and B. Z. Tang, Mechanochromic Luminescence of Aggregation-Induced Emission Luminogens, J. Phys. Chem. Lett., 2015, 6, 3429.

45 Z. Yang, Z. Chi, Z. Mao, Y. Zhang, S. Liu, J. Zhao, M. P. Aldred and Z. Chi, Recent advances in mechanoresponsive luminescence of tetraphenylethylene derivatives with aggregation-induced emission properties, Mater. Chem. Front., 2018, 2, 861.

46 R. T. K. Kwok, J. Geng, J. W. Y. Lam, E. Zhao, G. Wang, R. Zhan, B. Liu and B. Z. Tang, Water-soluble bioprobes with aggregation-induced emission characteristics for lightup sensing of heparin, J. Mater. Chem. B, 2014, 2, 4134.

47 C. W.-T. Chan, H.-K. Cheng, F. K.-W. Hau, A. K.-W. Chan and V. W.-W. Yam, Protamine-Induced Supramolecular Self-Assembly of Red-Emissive Alkynylplatinum(II) 2,6Bis(benzimidazol-2'-yl)pyridine Complex for Selective LabelFree Sensing of Heparin and Real-Time Monitoring of Trypsin Activity, ACS Appl. Mater. Interfaces, 2019, 11, 31585.

48 J. Zheng, T. Ye, J. Chen, L. Xu, X. Ji, C. Yang and Z. He, Highly sensitive fluorescence detection of heparin based on aggregation-induced emission of a tetraphenylethene derivative, Biosens. Bioelectron., 2017, 90, 245.

49 S. Li, M. Gao, S. Wang, R. Hu, Z. Zhao, A. Qin and B. Z. Tang, Light up detection of heparin based on aggregation-induced emission and synergistic counter ion displacement, Chem. Commun., 2017, 53, 4795. 